\title{
COUNTERPOINT Should minimal residual disease negativity not be the end point of myeloma therapy?
}

Pieter Sonneveld

Department of Hematology, Erasmus MC Cancer Institute, Rotterdam, The Netherlands

This article has a companion Point by Anderson.

\section{Background and introduction}

Multiple myeloma $(\mathrm{MM})$ is a malignant proliferation of clonal plasma cells in the bone marrow (BM). Its clinical course is characterized by the appearance of a monoclonal protein in serum and/or urine and symptomatic organ dysfunction such as renal impairment, osteolytic bone lesions, hypercalcemia, and anemia. Other diagnostic criteria include a high free serum $\kappa / \lambda$ ratio $\geq 100,>1$ focal lesion on magnetic resonance imaging (MRI), or $\geq 60 \%$ clonal plasma cells. ${ }^{1}$ Recently, the International Myeloma Working Group (IMWG) has defined revised criteria of response and minimal residual disease (MRD) for patients with $\mathrm{MM}^{2}$

During the last decade, several new treatment modalities have been introduced which have significantly improved the response rate and overall prognosis of $\mathrm{MM}^{3}$ Immunomodulatory agents and proteasome inhibitors are now standard treatment. ${ }^{4}$ An example of the high efficacy rate of the novel agents is a recent trial in relapsed/refractory $\mathrm{MM}$ (RRMM) in which carfilzomib combined with lenalidomide/ dexamethasone $(\mathrm{Rd})$ resulted in $31.8 \%$ complete response $(\mathrm{CR})$ vs $9.1 \%$ with $\mathrm{Rd}$ alone, whereas progression-free survival (PFS) was 26 vs 17 months. ${ }^{5}$ Also, ixazomib combined with Rd was superior to the backbone alone for CR and PFS. ${ }^{6}$

New active agents are the monoclonal antibodies daratumumab and isatuximab (anti-CD38), the checkpoint inhibitors durvalumab and nivolumab (anti-PD-1/PD1L), and elotuzumab (anti-SLAMF7). These agents alone or in combination with a backbone of $\mathrm{Rd}$ or bortezomib/dexamethasone have shown significant clinical activity in RRMM. ${ }^{7-10}$ Using these combinations, high overall response rates (ORRs) have been observed with significantly more CR, stringent $\mathrm{CR}$ (sCR), and longer PFS. Besides the addition of novel agents, the concept of clinical treatment has also changed significantly over the past years. For example, in younger patients, the following advances have contributed to improved response and/or PFS:

- Introduction of single or double high-dose therapy (HDT) and autologous stem cell transplantation in newly diagnosed and sensitive relapsed MM;

- Development of effective, triple drug-induction regimens prior to HDT including a proteasome inhibitor for 3 to 6 cycles;

- Use of consolidation treatment of 2 to 4 cycles after HDT;

- Use of maintenance therapy with lenalidomide or bortezomib alone or combined with corticosteroids. Several controlled trials have demonstrated that ORR and in particular CR or better improve with each subsequent treatment phase in newly diagnosed patients and hence should be monitored with highly sensitivity techniques.

\section{Response evaluation in MM}

The evaluation of response to therapy in $\mathrm{MM}$ has traditionally been based on the relative reduction of the $\mathrm{M}$ protein in serum and/or urine, the radiography of skeletal lesions, and the disappearance of elevated calcium, renal failure, anemia, and bone lesion (CRAB) symptomatology. Over time, new response levels such as verygood partial response, sCR, and serum free light chain value have been introduced in an attempt at standardization across clinical trials and in clinical practice. BM assessment of plasma cells ( $<5 \%$ for CR, no clonal plasma cell population for $\mathrm{SCR}$ ) has been a cornerstone for best response. In addition, the recent improvement in ORR and also the depth of response in a significant proportion of patients has again raised the question of whether a good response reliably predicts longer survival. Several groups have pioneered the concept of MRD and some analyses indicate that MRD negativity predicts for prolonged survival. ${ }^{11-15}$ Recently, the IMWG has revised the criteria for response and included MRD (Table 1). These revisions were 


\begin{tabular}{|c|c|c|c|}
\hline End point & Assay & Disadvantage & Target group(s) \\
\hline \multirow[t]{6}{*}{$\mathrm{CR} / \mathrm{sCR}$} & Standard technology & Less sensitive & NDMM elderly/frail \\
\hline & Benchmark in clinical trial & & RRMM \\
\hline & Widely applicable & & \\
\hline & Evidence based & & \\
\hline & No extra costs & & \\
\hline & No need for central laboratory & & \\
\hline \multirow[t]{5}{*}{ MRD by MFC } & Represents immunophenotypic response & Requires processing within $48 \mathrm{~h}$ & Transplant-eligible NDMM \\
\hline & Sensitivity $\geq 10^{-4}$ & Requires $\geq 5 \times 10^{6}$ cells & Clinical trials \\
\hline & Standard Euroflow protocol & Cost of assay & \\
\hline & Automated analysis & Less sensitive than NGS & \\
\hline & Available in many centers & & \\
\hline \multirow[t]{4}{*}{ MRD by qPCR } & Represents molecular response & Labor intensive & Transplant-eligible NDMM \\
\hline & Sensitivity $\geq 10^{-5}$ & Requires $\geq 10^{6}$ cells & Clinical trials \\
\hline & Standardized in ALL and other diseases & Applicable in $\leq 70 \%$ of patients & \\
\hline & Widely available & Requires baseline sample & \\
\hline \multirow[t]{5}{*}{ MRD by VDJ NGS } & Sensitivity $\geq 10^{-5}$ & Labor intensive & Transplant-eligible NDMM \\
\hline & Commercial product by $1-2$ companies & Requires $\geq 10^{6}$ cells & Clinical trials \\
\hline & & Applicable in $\leq 90 \%$ of patients & \\
\hline & & Requires bioinformatics & \\
\hline & & Requires baseline sample & \\
\hline \multirow[t]{2}{*}{ (MRD+)PET-CT } & Evaluation of extramedullary disease & Standard not yet defined as in lymphomas & All \\
\hline & Widely available & & \\
\hline \multirow[t]{3}{*}{ MRD by CTCs/RNA/DNA } & Assay in blood & Requires single-cell NGS & All \\
\hline & No sampling error & Not widely available & \\
\hline & Represents whole-body tumor mass & Not standardized & \\
\hline
\end{tabular}

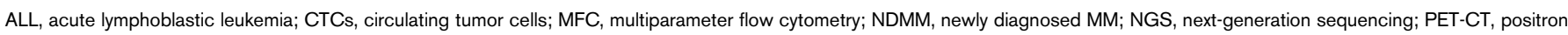
emission tomography-computed tomography; qPCR, quantitative PCR.

made because several studies indicated that patients who achieve CR and remain MRD-positive have worse PFS than patients who are MRDnegative. ${ }^{16,17}$ The IMWG guideline recognizes 4 MRD-based response criteria (eg, sustained MRD-negative, flow MRD-negative, sequencing MRD-negative, and imaging plus MRD-negative), which have been added to the classical response criteria. ${ }^{2} \mathrm{~A}$ recent metaanalysis has confirmed that MRD-negative disease status is associated with prolonged survival. ${ }^{18}$

Should MRD negativity not be the standard end point of MM therapy?

In order to answer this question, the pros and cons of MRD assessment will be summarized.

- Why MRD? With CR rates approaching 60\% and more, including $30 \%$ to $40 \%$ sCR using novel agents in transplant-eligible NDMM, new definitions of good responses and quantitation of the remaining tumor burden are needed in order to identify relevant prognostic subgroups of patients. Typically, patients in clinical trials should be evaluated and followed with MRD, whereas in general practice this is not (yet) the standard.

- When should MRD be done or not? MRD assessment may be a useful tool in treatment situations where the expected frequency of $\mathrm{CR} / \mathrm{sCR}$ is high. However, in non-transplant-eligible patients as well as patients with RRMM, this will not likely be the case and few patients achieve a durable CR. In particular, attenuated treatment schedules in the elderly/frail rarely result in high $\mathrm{CR}$ rates and treatment choices are primarily decided by toxicity rather than to achieve MRD negativity. In those patients, conventional response criteria as defined by IMWG may suffice.

- Is there a standard assay for MRD? In the current era, MRD assays are based on MFC using 8 to 10 colors or on NGS. ${ }^{12,15,19}$ The sensitivity of MFC is $10^{-4}$ to $10^{-5}$ and this type of response has been defined as immunophenotypic response. However, samples need to be handled fresh by a designated laboratory using defined protocols, and the interpretation of the results is subjective. NGS is more sensitive $\left(10^{-4}\right.$ to $\left.10^{-6}\right)$ and can be done on frozen samples. Results typically become available within weeks. MRD by NGS represents the molecular response. At this moment, there is no unanimous preference for either technique and, because they represent different outcomes, MRD comparisons and analyses between trials is difficult. In addition, although consensus guidelines are lacking for time points of MRD, technical harmonization, value of MFC vs NGS or polymerase chain reaction (PCR) MRD negativity remains a subjective end point 
which is useful only in the context of the specific trial where it is investigated.

- Does MRD represent the true remission status? Assessment of MRD is performed on material obtained from BM aspirates. It is well known that $\mathrm{MM}$ is a patchy disease with areas of tumor cells amid normal hematopoiesis. The heterogeneous pattern of distribution may generate false-negative punctures and inadequate MRD results. Although this may be less of a problem when looking for absence of clonal plasma cells as in SCR, it is troublesome for accurate quantification of the tumor load at levels of $10^{-4}$ to $10^{-6}$. In addition, there may be sanctuaries with surviving refractory tumor cells or extramedullary disease that have escaped from previous therapy. Because the ultimate goal of MRD is to achieve a quantitative tumor assessment on which future treatment choices can be based, one may challenge the current technique of taking a snapshot sample. In such cases, MRD negativity underestimates the true presence of remaining, refractory tumor cells that have escaped from previous therapy.

- When MRD? At this moment, MRD has mainly been assessed in transplant-eligible patients after HDT. Few data are available for systematic sequential analysis during later stages of treatment. It is not clear what the optimal time points for analysis are and how long patients should be followed after obtaining MRD negativity.

- Is BMMRD sufficient? Several groups have attempted to improve the current MRD approach by including imaging techniques, specifically PET-CT and/or MRI. ${ }^{20}$ These initiatives have demonstrated that PET-CT-positive lesions frequently occur in MRDnegative patients and that PET-CT negativity is associated with better PFS. Consequently, PET-CT-positive lesions need to be punctured to confirm (treatment-refractory) MM. This further illustrates the shortcomings of snapshot MRD analysis in BM.

- Will patients accept repetitive BM punctures? BM punctures are painful procedures that are not acceptable for all patients. Given the current dilemmas with sampling errors and choice of technique, other diagnostic innovations may be considered. One of these is the analysis of CTCs or micro-RNAs in blood. ${ }^{21,22}$ CTCs may originate from any tumor site in the body and thus represent the overall disease situation. Efforts are needed to develop quantitative assays to detect CTCs using a NGS-based approach.

- The economic impact of MRD analysis. The estimated cost of a single MRD noncommercial analysis by MFC (10 colors) is $\$ 500$ and by NGS $\$ 350$. Given the frequency of inadequate punctures, failures by NGS (10\%), transport, and business models, the average cost will be significant. These expenses come on top of high drug prices and can only be justified if the results are used for rational treatment decisions.

Until now, MRD negativity has not yet been accepted as the main end point for clinical trials by the regulatory authorities and discussions are ongoing. The current data were obtained in prospective clinical trials which only included selected patients who were treated with intensive regimens and were carefully monitored for all aspects of their disease. In that particular context, the data suggest that MRD is a useful prognostic marker for PFS in transplant-eligible NDMM. However, the value of MRD should also be evaluated in prospective clinical trials with less intensive regimens and in less favorable subgroups of patients such as non-transplant-eligible MM, frail elderly patients, and RRMM. In (elderly) patients who continue on maintenance therapy, MRD assessment may help to decide on the duration of treatment. Only if MRD negativity has a better prognostic impact than CR in those patients may it be routinely used.

More importantly, as yet there are no data which prove that MRD positivity or negativity may influence treatment decisions which have an effect on survival. Prospective trials have to be designed in order to conclude whether the use of MRD negativity for treatment choices contributes to an improvement of long-term outcome such as overall survival. Once these data become available, efforts have to be made to evaluate the possible application of MRD in individual patients outside of the context of clinical trials. The coming years will reveal whether MRD will be included as a standard evaluation in treatment algorithms for first and later lines of therapy.

\section{Authorship}

Contribution: P.S. wrote the paper and approved the final version of the paper.

Conflict-of-interest disclosure: P.S. received honoraria from Janssen, Celgene, Amgen, Karyopharm, and Takeda.

Correspondence: Pieter Sonneveld, Department of Hematology, Erasmus MC Cancer Institute, Room Na822, Wytemaweg 80, 3015 CN Rotterdam, The Netherlands; e-mail: p.sonneveld@erasmusmc.nl.

\section{References}

1. Rajkumar SV, Dimopoulos MA, Palumbo A, et al. International Myeloma Working Group updated criteria for the diagnosis of multiple myeloma. Lancet Oncol. 2014;15(12):e538-e548.

2. Kumar S, Paiva B, Anderson KC, et al. International Myeloma Working Group consensus criteria for response and minimal residual disease assessment in multiple myeloma. Lancet Oncol. 2016;17(8):e328-e346.

3. Palumbo A, Anderson K. Multiple myeloma. N Engl J Med. 2011;364(11):1046-1060.

4. Palumbo A, Rajkumar SV, San Miguel JF, et al. International Myeloma Working Group consensus statement for the management, treatment, and supportive care of patients with myeloma not eligible for standard autologous stem-cell transplantation. J Clin Oncol. 2014;32(6):587-600.

5. Stewart AK, Rajkumar SV, Dimopoulos MA, et al; ASPIRE Investigators. Carfilzomib, lenalidomide, and dexamethasone for relapsed multiple myeloma. N Engl J Med. 2015;372(2): 142-152.

6. Moreau P, Masszi T, Grzasko N, et al; TOURMALINE-MM1 Study Group. Oral ixazomib, lenalidomide, and dexamethasone for multiple myeloma. N Engl J Med. 2016;374(17):1621-1634.

7. Lonial S, Dimopoulos M, Palumbo A, et al; ELOQUENT-2 Investigators. Elotuzumab therapy for relapsed or refractory multiple myeloma. N Engl J Med. 2015;373(7):621-631.

8. Lokhorst HM, Plesner T, Laubach JP, et al. Targeting CD38 with daratumumab monotherapy in multiple myeloma. $N$ Engl $J$ Med. 2015;373(13):1207-1219.

9. Palumbo A, Chanan-Khan A, Weisel K, et al; CASTOR Investigators. Daratumumab, bortezomib, and dexamethasone for multiple myeloma. N Engl J Med. 2016;375(8):754-766. 
10. Dimopoulos MA, Oriol A, Nahi H, et al; POLLUX Investigators. Daratumumab, lenalidomide, and dexamethasone for multiple myeloma. N Engl J Med. 2016;375(14):1319-1331.

11. Paiva B, Vidriales MB, Cerveró J, et al; GEM (Grupo Español de MM)/PETHEMA (Programa para el Estudio de la Terapéutica en Hemopatías Malignas) Cooperative Study Groups. Multiparameter flow cytometric remission is the most relevant prognostic factor for multiple myeloma patients who undergo autologous stem cell transplantation. Blood. 2008; 112(10):4017-4023.

12. Rawstron AC, Child JA, de Tute RM, et al. Minimal residual disease assessed by multiparameter flow cytometry in multiple myeloma: impact on outcome in the Medical Research Council Myeloma IX Study. J Clin Oncol. 2013;31(20):2540-2547.

13. Ladetto $M$, Omedè $P$, Sametti $S$, et al. Real-time polymerase chain reaction in multiple myeloma: quantitative analysis of tumor contamination of stem cell harvests. Exp Hematol. 2002; 30(6):529-536.

14. Landgren $\mathrm{O}$, Gormley $\mathrm{N}$, Turley $\mathrm{D}$, et al. Flow cytometry detection of minimal residual disease in multiple myeloma: Lessons learned at FDA- $\mathrm{NCl}$ roundtable symposium. Am J Hematol. 2014;89(12):1159-1160.

15. Paiva B, van Dongen JJ, Orfao A. New criteria for response assessment: role of minimal residual disease in multiple myeloma. Blood. 2015;125(20):3059-3068.

16. Attal M, Lauwers-Cances V, Marit G, et al; IFM Investigators. Lenalidomide maintenance after stem-cell transplantation for multiple myeloma. N Engl J Med. 2012;366(19):1782-1791.
17. Paiva B, Gutiérrez NC, Rosiñol L, et al; PETHEMA/GEM (Programa para el Estudio de la Terapéutica en Hemopatías Malignas/Grupo Español de Mieloma) Cooperative Study Groups. High-risk cytogenetics and persistent minimal residual disease by multiparameter flow cytometry predict unsustained complete response after autologous stem cell transplantation in multiple myeloma. Blood. 2012;119(3):687-691.

18. Munshi NC, Avet-Loiseau H, Rawstron AC, et al. Association of minimal residual disease with superior survival outcomes in patients with multiple myeloma: a meta-analysis. JAMA Oncol. 2017;3(1):28-35.

19. Munshi NC, Anderson KC. Minimal residual disease in multiple myeloma. J Clin Oncol. 2013;31(20):2523-2526.

20. Zamagni E, Nanni C, Mancuso K, et al. PET/CT improves the definition of complete response and allows to detect otherwise unidentifiable skeletal progression in multiple myeloma. Clin Cancer Res. 2015;21(19):4384-4390.

21. Gonsalves WI, Morice WG, Rajkumar V, et al. Quantification of clonal circulating plasma cells in relapsed multiple myeloma. Br J Haematol. 2014;167(4):500-505.

22. Kubiczkova L, Kryukov F, Slaby O, et al. Circulating serum microRNAs as novel diagnostic and prognostic biomarkers for multiple myeloma and monoclonal gammopathy of undetermined significance. Haematologica. 2014;99(3): 511-518.

DOI 10.1182/bloodadvances. 2017000109

(C) 2017 by The American Society of Hematology 